\title{
QCD PROCESSES AT THE LHC
}

\author{
GEORGIOS D. STAVROPOULOS \\ CERN
}

\begin{abstract}
The study of QCD processes at the LHC will serve two main goals. First the predictions of QCD will be tested and precision measurements will be performed. Second QCD processes represent a major part of the background to other Standard Model processes and signals of new physics at the LHC and thus need to be understood precisely in the new kinematic region available here. Furthermore, the production cross-sections for almost all processes are controlled by QCD.
\end{abstract}

\section{Introduction}

The Large Hadron Collider (LHC) is a proton-proton collider with $14 \mathrm{TeV}$ center of mass energy and design luminosity of $10^{34} \mathrm{~cm}^{-2} \mathrm{~s}^{-1}$. Beam crossings are $25 \mathrm{~ns}$ apart and at design luminosity there are on average 23 interactions per crossing. $10 \mathrm{fb}^{-1}$ of integrated luminosity are expected to be collected in one year of data taking at the initial low $\left(10^{33} \mathrm{~cm}^{-2} \mathrm{~s}^{-1}\right)$ luminosity and 100 $\mathrm{fb}^{-1}$ for one year of data taking at the nominal one.

A detailed understanding of QCD is important for almost all the physics processes to be studied at the LHC, as the production mechanisms are mostly controlled by QCD. LHC will allow QCD studies to be performed at very high energy, including precision tests and measurements in an as yet unexplored kinematic region.

\section{Jet Physics}

The measurement of jet production cross-section at LHC will provide a stringent test of perturbative QCD in an energy regime never probed so far. In Figure 1 $1(a)$ the inclusive jet cross-section 1 is shown as a function of the transverse energy of the jet, $E_{T}$, for three different bins in $\eta$. The expected statistics for an integrated luminosity of $30 \mathrm{fb}^{-1}$ amounts to $4 \cdot 10^{5}$ events with $E_{T}^{\text {jet }}>$ $1 \mathrm{TeV}$, demonstrating the fact that at LHC the statistical uncertainties on the jet cross-section measurement will be small. More important in this measurement are the systematic errors, which can arise from the jet algorithm, the knowledge of the jet energy scale and the understanding of the overall calorimeter response, the knowledge of the jet trigger efficiency, the knowledge of the luminosity for the overall normalization, and the effect of the underlying event. The expected statistics at large jet $E_{T}$ values implies a 

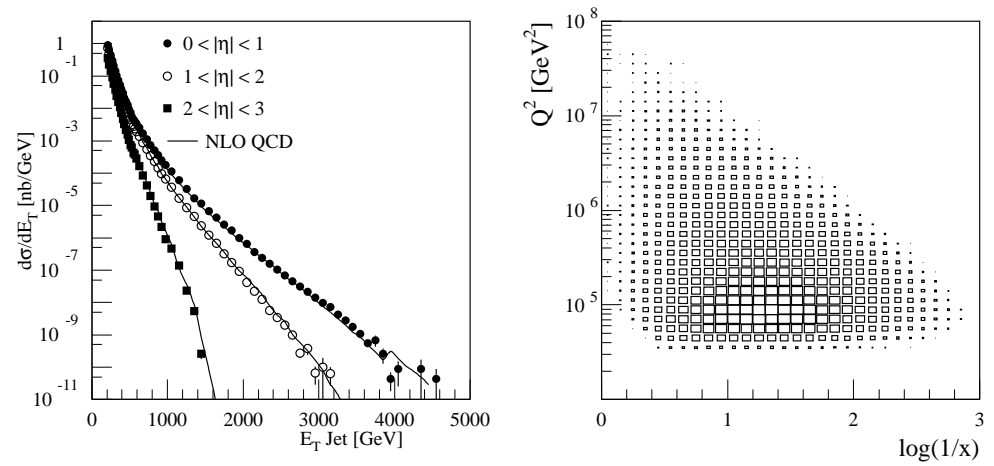

Figure 1. (a): Inclusive jet cross-section as a function of the transverse energy of the jet. (b): Range in $1 / x$ and $Q^{2}$ for the di-jet differential cross-section measurement.

desirable control of the systematic uncertainties to a precision of better than $1 \%$ for $E_{T}<1 \mathrm{TeV}$ and to about $10 \%$ for $E_{T}$ of about $3 \mathrm{TeV}$. The jet crosssection measurement can be used for the determination of $\alpha_{s}$. It was shown that at LHC a measurement of $\alpha_{s}\left(M_{Z}\right)$ up to scales of order TeV can be achieved with a $10 \%$ accuracy.

The measurement of di-jet events and their properties can be used to constrain the parton densities inside the proton, in various kinematic regions of $Q^{2}$ and $x$. In Figure $1(b)$ the expected range in $x$ and $Q^{2}$ accesible with the di-jet differential cross-section measurement is shown. For a transverse energy threshold of $180 \mathrm{GeV}$, most of the events have $Q^{2}$ values of about $10^{5}$ and values of $x$ between $2 \cdot 10^{-3}$ and 0.5 .

The di-jet invariant mass and angular distributions can be used to search for new physics. Studies have shown that the di-jet angular distribution has an excellent discovery capability for quark compositeness. An integral luminosity of $300 \mathrm{fb}^{-1}$ will allow excluding quark substructure at $95 \% \mathrm{CL}$, if the constituent interaction constant is of the order of $40 \mathrm{TeV}$ (see figure 2(a)).

\section{Photon Physics}

Direct photon measurements can provide important constraints on parton distributions, especially on the gluon distribution in the proton. The detection of photons at hadron collider is a challenging task due to the large background from jet production, where fluctuations can mimic the signature of a photon. 

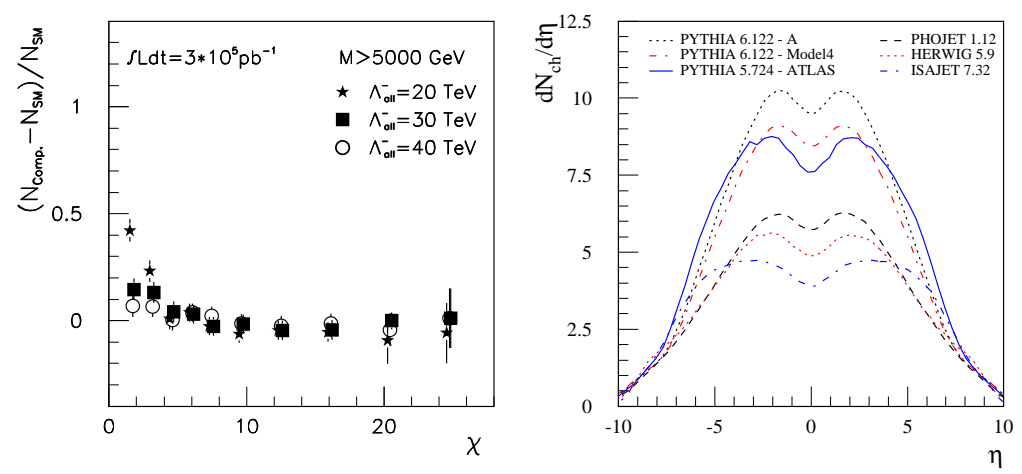

Figure 2. (a): Deviation of the di-jet angular distribution from the Standard Model predictions, for di-jet mass above $5 \mathrm{TeV}$ for $300 \mathrm{fb}^{-1}$. (b): Charged particle density in minimumbias events at LHC energies as a function of pseudorapidity, for six model predictions.

As an example in ATLAS it was found that for a photon efficiency of $80 \%$ a rejection factor against jets of larger than $3 \times 10^{3}$ can be achieved for $E_{T}>$ $40 \mathrm{GeV}$, thus providing a good signal-to-background ratio. The selection at ATLAS will be based on a trigger demanding an isolated photon of $E_{T}>60$ $\mathrm{GeV}$ within $|\eta|<2.5$. This gives a value of the Bjorken- $x$ of $5 \cdot 10^{-4}$.

\section{Lepton Physics}

The measurement of the Drell-Yan lepton pair production and the production of $W$ and $Z$ bosons (with a leptonic decay to electrons or muons) will allow one to constrain the quark and anti-quark densities of the proton at a scale given by the invariant mass of the lepton pair ( $W / Z$ mass respectively) over a wide range in Bjorken- $x$. For example, the measurement of $Z$ boson production cross-section allows one to cover the range of $3 \cdot 10^{-4}<x<0.1$ in Bjorken- $x$ at $Q^{2}=8 \cdot 10^{3} \mathrm{GeV}^{2}$.

\section{$5 \quad$ Hard Diffractive Scattering}

During the last few years the understanding of diffractive phenomena has received revived attention due to the observation of hard diffractive events in several experiments. Thus, the study of hard diffractive scattering at LHC becomes an important topic of the physics program of the involved exper- 
iments. An extension of the LHC detectors in the forward region (beyond pseudo-rapidities of $|\eta|=5$ ) is presently under study, in order to increase the acceptance for charged particles from inelastic interactions (possibly including a momentum measurement) and to provide tagging and measurement of leading protons from elastic and diffractive interactions.

\section{Properties of minimum-bias events}

At the LHC, minimum-bias events will make up the 23 interactions per bunch crossing at high luminosity. In order to understand precisely their contribution to the measured quantities for the hard scattering events of interest, a detailed knowledge of the structure of the minimum-bias events is required. In Figure 2 (b) the charged particle density in minimum-bias events at LHC energies is shown as a function of pseudorapidity. Six models, tuned on CDF data, were used for this calculation. There is a difference of a factor 2 in the expected charged particle density in the central region, for models which are able to describe CDF data. Each minimum-bias event is expected to contribute from charged particles about 0.5 to $1 \mathrm{GeV}$ per unit rapidity and unit azimuth to the transverse energy.

\section{Conclusions}

The LHC will provide a large sample of events with high $p_{T}$ signatures for QCD studies. It will extend the kinematic range to values of $Q^{2}$, the hard scale of the partonic process, of the order of $\mathrm{TeV}^{2}$. The fraction of the proton momentum attributed to a parton will reach values below $10^{-5}$, while keeping the hard scattering scale above $100 \mathrm{GeV}^{2}$.

A precise knowledge and understanding of QCD processes is important for the studies of the Higgs boson(s) and for searches for new physics beyond the Standard Model, where QCD represents a large part of the background.

\section{References}

1. ATLAS Detector and Physics Performance Technical Design Report, CERN/LHCC 99-14 and 99-15 (1999).

2. H. Stenzel, Determination of $\alpha_{s}$ using jet cross-section parameterizations at hadron colliders, ATL-PHYS-2001-003, March 26, 2001. 